1000. $83 \%$ of the services did not have an audiometer or spirometer, $86 \%$. Only $56 \%$ of the services met the threshold of audibility during the pre-occupational evaluation. $76.6 \%$ of workers who moved weights had a lumbar X-ray. A significant proportion could not maintain professional secrecy, due to company requirements. The opinion of the workers contrasted with the opinion of the medical services. Only 19\% of the services delivered the results of the evaluations to the workers. $52.38 \%$ considered that the medical service kept professional secrecy.

Conclusions There was a low detection and notification of occupational diseases, little equipment of services for preventive activities, and it is necessary to ensure that the medical service has the power to keep medical secrecy.

\section{P-387 SUCCESS OF REHABILITATION AMONG 185.385 EMPLOYEES UNDERGOING MEDICAL REHABILITATION FROM 2010 TO 2012 IN GERMANY.}

${ }^{1}$ Maria Weyermann, Nicole Wist. 'Niederrhein University of Applied Sciences, Germany

\subsection{6/OEM-2021-EPI.304}

Introduction In Germany the statutory pension insurance fund covers the cost of rehabilitation treatment for employees whose working capacity is endangered due to health problems.

Objectives We aimed to describe success of medical rehabilitation (work ability at discharge, return to work in the year after rehabilitation, early retirement within seven years after rehabilitation) with special consideration of potential sociodemographic determinants among employed persons undergoing medical rehabilitation from 2010 to 2012 in Germany.

Methods Analysis based on Scientific Use Files of administrative pension records from the Research Data Centre of the German Federal Pension Insurance, which include 20 percent random samples of all cases of medical rehabilitation. Risk of low work ability at discharge (LWA) and failed return to work in the year after rehabilitation (FRW) was estimated using logistic regression models, risk of early retirement during seven years after rehabilitation (ER) was estimated using cox proportional hazard models. Age, sex, citizenship, school and vocational education, annual income before rehabilitation and medical diagnosis were considered as potential risk factors.

Results We included 185.385 employed persons aged 18 to 60 years undergoing medical rehabilitation from 2010 to 2012. Low work ability at the end of rehabilitation was reported among $7.9 \%, 26 \%$ showed unsufficient return to work one year after rehabilitation, and $9.7 \%$ received disability pension during 7-year follow-up, respectively. In fully adjusted models, non-German citizenship, older age, low educational level, as well as low annual income were risk factors for each outcome of work ability. For example, adjusted risk among persons with low annual income (1st quartile vs. 4th quartile, OR/HR [95\%-CI]) was $2.3[2.2 ; 2.4]$ for FRW, 4.8 [4.5; 5.1] for LWA, and $2.5[2.3 ; 2.6]$ for ER.

Conclusions These results continue to show that success of medical rehabilitation is lower in deprived social groups in Germany.

\section{P-389 RETURN TO WORK AFTER ACUTE MYOCARDIAL INFARCTION: A COMPARISON BETWEEN YOUNG ACTIVE WOMEN AND MEN}

${ }^{1}$ Amira Omrane, Olfa Jlassi, Majdi Ben Massoud, Majed Hassine, Lamia Bouzgarrou, Taoufik Khalfallah, Habib Gamra. ${ }^{1}$ Faculty of Medicine of Monastir, Tunisia

\subsection{6/OEM-2021-EPI.305}

Background Return to work (RTW) after an acute myocardial infarction (AMI) is an important outcome particularly relevant to young and active patients. Women may be at a greater risk for not returning to work given evidence of their worse recovery after AMI than similarly aged men. However, sex differences in return to work after AMI has not been studied extensively in a young active population ( $\leq 65$ years). This study aimed to assess return to work among patients after an AMI and evaluate the role of gender.

Methods We prospectively followed patients with a first time AMI, employed at the time of the index hospitalization between June 2018 and December 2019, in the Cardiology department in a Public hospital in the central region of Tunisia. Data were obtained by medical record abstraction and patient interviews. Data collected were related to socio-demographic characteristics, clinical characteristics and the validated MacNew Questionnaire.

Results Fifty patients were enrolled aged 35 to 65 years. The study group was made up of $45(90 \%)$ male and $5(10 \%)$ female. Patients who did and did not return to work did not differ in gender distribution $(p=0.79)$. Women returned to work later than men (respectively after 65 and 75 days) without statistically significant difference $(p=0.87)$. Eighty four percent of the study population returned to work within six months. Smoking, left ventricular ejection, Killip class, in-hospital complications, global physical activity and Quality of Life were found to be significantly associated with return to work in independent univariate analyzes. A stepwise multivariate regression analysis identified only the Quality of Life and the number of children in charge as predictors of RTW.

Conclusion This study revealed a higher probability of RTW in males among a young active population. This difference is explained by disparities in demographic, occupational and health characteristics.

\section{P-390 USING A CLINICAL DATABASE TO INFORM PREVENTION OF OCCUPATIONAL SKIN DISEASE WITH A FOCUS ON THE HEALTH CARE SECTOR.}

'D Linn Holness, Sandy Skotnnicki, Joel DeKoven, Irena Kudla. 'University of Toronto, Canada and St Michael's Hospital, Canada

\subsection{6/OEM-2021-EPI.306}

Introduction Clinical databases provide useful information on occupational diseases including occupational skin diseases. Patch testing is an important tool in the diagnosis of occupational contact dermatitis. Patch test databases not only contain specific diagnostic information but also identify affected occupations and workplace characteristics allowing comparisons across industries and jobs. Additional workplace information can be added to gain insight into prevention activities. 
Objectives To examine the diagnosis, common workplace allergens and prevention practices in workers seen for patch testing in a tertiary referral centre in Toronto, Canada.

Methods Demographic, clinical, patch test and workplace information were collected for patients seen between 2012 and 2019. Basic descriptive statistics were generated to compare workers in common industries and jobs.

Results Out 3714 patients evaluated, 1261 were diagnosed with occupational skin disease. Comparison across the healthcare, services, manufacturing, automotive and construction sectors revealed differences in diagnosis and causative agents, workplace characteristics and prevention practices. 308 health care workers included 154 nurses, 34 personal support workers, 28 dental workers and 22 cleaners. 90\% had a diagnosis of occupational irritant contact dermatitis and 34\% had occupational allergic contact dermatitis. Dental workers had the highest percentage of allergic contact dermatitis and higher proportions of occupationally relevant rubber (carba mix and thiuram) and methacrylate/acrylate positives on patch testing. They were more likely to work in a small workplace and less likely to take time off work, file a compensation claim or have health and safety training.

Conclusion Collection of detailed work-related descriptors and clinical information in a patch test database facilitates an understanding of the causative agents and the workplace characteristics that may place workers at increased risk for occupational skin disease, providing a focus for prevention activities.

\section{P-393 HAND AND WRIST MUSCULOSKELETAL DISORDERS ASSOCIATED WITH BIOMECHANICAL RISK FACTORS AMONG TUNISIAN WEAVERS}

${ }^{1}$ Asma Kheder, Ben Afia Latifa, Faten Hermassi, Amira Omrane, Taoufik Khalfallah, Lamia Bouzgarrou. 'University of Monastir - School of Medicine, Tunisia

\subsection{6/OEM-2021-EPI.307}

Introduction The informal industry of handmade carpet is particularly developed in the Tunisian central region with use of ancestral tools. The hand and the wrist are continually solicited during the different performed tasks, which may result in musculoskeletal disorders.

Objective This study aimed to assess the biomechanical constraints of the wrist and hand among Tunisian weavers.

Methods A 6 months study was conducted among a representative sample of traditional weavers in a region of central Tunisia. The survey was based on semi-quantitative ergonomic assessment of biomechanical risk factors, with video recordings during a period of full activity (25 to 40 minutes). Each recording was stopped at fixed time intervals and converted into one hundred images. For each image, the postures of the wrists and hands were encoded and analyzed with ERGOROM software. The Gestural Variability Score (GVS) and the subjective BORG scale were used to estimate postural variation and the level of exerted force.

Results The average duration of the working day was $7.45 \pm$ $2.5 \mathrm{~min}$. The weavers spent $57.5 \%$ of the working time with a wrist extension $>30^{\circ}$ with a relatively high Flexion/Extension GVS of $46 \%$. In the coronal plane, radial and ulnar deviation were found during $11.5 \%$ and $9.4 \%$ of the activity time, respectively. Pinching grip of hand tools was spread over $83.4 \%$ of the working time range with a relatively low GVS (22.2\%). According to the BORG scale, $78.5 \%$ of the weavers estimated the force exerted by the hands and wrists as 'medium' to 'strong' during the weaving activity.

Conclusion During artisanal weaving, the hand and the wrist are deviated from the neutral position adopting awkward postures. Moreover, they are exposed to forceful gripping and repetitive movement, which may lead to musculoskeletal injuries. The prevention of these damages should focus on rethinking poorly designed hand tools.

\section{P-394 MUSCULOSKELETAL DISORDERS AMONG MILITARY DENTISTS: SEMI-QUANTITATIVE ERGONOMIC RISK ASSESSMENT}

${ }^{1}$ Kamoun Salma, Olfa Jlassi, Lamia Bouzgarrou, Jilani Gnounou, Adel Amri. ${ }^{1}$ Centre Militaire de Médecine du Travail et de Sécurité Professionnelle, Tunisia

\subsection{6/OEM-2021-EPI.308}

Introduction The activity of the dentist, notably in the military force, is faced like many others the scourge of musculoskeletal disorder (MSD).

Objectives This study aimed to identify the MSD hazards among dentists and identify work situations that can contribute to their appearance and offer appropriate ergonomic remedial measures for their prevention.

Methods This is a semi-quantitative study conducted in a military dental surgery service in Tunis by observing biomechanical constraints during three different dental acts. These observations were then analysed using ERGOROM software.

Results The MSD hazards identified in this study as common to all dentists were strained postures, repetition, static shoulder postures and bad working position. The neck was in more than 40 degrees flexion or extended over $63 \%$ to $95 \%$ of the working time. The shoulders were in sustained contraction over 40 to $72 \%$ of the working time with a low variation suggesting an isometric shoulder contraction. The elbows were in flexion over 56 to $91 \%$ of the working time with low variation flexion/extension. The preferred wrist position was hyper-extension or hyper-flexion over 20 to $64 \%$ of working time and ulnar or radial deviation over 14 to $35 \%$ of working time. Isometric dental instruments grasp spread over almost the whole working time for the three observed dental acts.

Conclusion Our study confirmed biomechanical constraints and MSD hazards to which dentists are exposed and guided us in our preventive approach in order to propose the most adequate and appropriate solutions to the military command.

\section{P-395 WORKPLACE BULLYING AMONG HEALTHCARE WORKERS: PREVALENCE AND IMPACT ON MENTAL HEALTH}

${ }^{1}$ Asma Kheder, Amen Moussa, Naïma Bouattay, Harrathi Chayma, Lotfi Gaha, Taoufik Khalfallah, Ahmed M'halla, Lamia Bouzgarrou. ' University of Monastir - School of Medicine, Tunisia

\subsection{6/OEM-2021-EPI.309}

Introduction Workplace bullying (WPB) is a peculiar form of workplace violence. This behavior affects the healthcare sector in general and particularly intensive care units such as anaesthesiology. 